Shockwave Medicine 


\title{
Translational Research in Biomedicine
}

\section{Vol. 6}

Series Editor

Samuel H.H. Chan Kaohsiung

\author{
Associate Editor \\ Julie Y.H. Chan Kaohsiung
}

The Chang Gung Medical Foundation is the patron of this book series. 


\section{Shockwave Medicine}

Volume Editors

Ching-Jen Wang kaohsiung

Wolfgang Schaden vienna

Jih-Yang Ko kaohsiung

17 figures, 11 in color, 9 tables, 2018

KARGER 


\section{Ching-Jen Wang}

Center for Shockwave Medicine and Tissue

Engineering, Department of Orthopedic

Surgery, Kaohsiung Chang Gung Memorial Hospital and Chang Gung University College of

Medicine

123, Ta-Pei Road

Kaohsiung 83301

Taiwan, ROC

\section{Wolfgang Schaden}

Ludwig Boltzmann Institute for Experimental and Clinical Traumatology

Donaueschingenstraße 13

A-1200 Vienna

Austria

Library of Congress Cataloging-in-Publication Data

\section{Jih-Yang Ko}

Center for Shockwave Medicine and Tissue

Engineering, Department of Orthopedic

Surgery, Kaohsiung Chang Gung Memorial

Hospital and Chang Gung University College of

Medicine

123, Ta-Pei Road

Kaohsiung 83301

Taiwan, ROC

Names: Wang, Ching-Jen, 1939- editor. | Schaden, Wolfgang, editor. | Ko, Jih-Yang, 1955- editor.

Title: Shockwave medicine / volume editors, Ching-Jen Wang, Wolfgang Schaden, Jih-Yang Ko.

Other titles: Translational research in biomedicine ; v. 6. 1662-405X

Description: Basel ; New York : Karger, 2018. | Series: Translational research in biomedicine, ISSN 1662-405X; vol. 6 | Includes bibliographical references and indexes.

Identifiers: LCCN 2018001691| ISBN 9783318063127 (hardcover : alk. paper) |

ISBN 9783318063134 (electronic version)

Subjects: |MESH: Extracorporeal Shockwave Therapy

Classification: LCC RC483.9 | NLM WB 515 | DDC 616.89/122--dc23 LC record available at

https://lccn.loc.gov/2018001691

Disclaimer. The statements, opinions and data contained in this publication are solely those of the individual authors and contributors and not of the publisher and the editor(s). The appearance of advertisements in the book is not a warranty, endorsement, or approval of the products or services advertised or of their effectiveness, quality or safety. The publisher and the editor(s) disclaim responsibility for any injury to persons or property resulting from any ideas, methods, instructions or products referred to in the content or advertisements.

Drug Dosage. The authors and the publisher have exerted every effort to ensure that drug selection and dosage set forth in this text are in accord with current recommendations and practice at the time of publication. However, in view of ongoing research, changes in government regulations, and the constant flow of information relating to drug therapy and drug reactions, the reader is urged to check the package insert for each drug for any change in indications and dosage and for added warnings and precautions. This is particularly important when the recommended agent is a new and/or infrequently employed drug.

All rights reserved. No part of this publication may be translated into other languages, reproduced or utilized in any form or by any means electronic or mechanical, including photocopying, recording, microcopying, or by any information storage and retrieval system, without permission in writing from the publisher.

(c) Copyright 2018 by S. Karger AG, P.O. Box, CH-4009 Basel (Switzerland)

www.karger.com

Printed on acid-free and non-aging paper (ISO 9706)

ISSN 1662-405X

e-ISSN 1662-4068

ISBN 978-3-318-06312-7

e-ISBN 978-3-318-06313-4 


\section{Contents}

\section{Foreword}

Chan, S.H.H. (Kaohsiung)

viII Preface

Wang, C.-J. (Kaohsiung); Schaden, W. (Vienna); Ko, J.-Y. (Kaohsiung)

Section I: Introduction

1 History of Shockwave Treatment and Its Basic Principles

Vincent, K.C.S. (Auckland/Victoria); d'Agostino, M.C. (Milan)

Section II: Musculoskeletal Disorders

17 Development of Extracorporeal Shockwave Therapy for Treatment of Osteonecrosis of the Femoral Head

Cheng, J.-H.; Hsu, S.-L.; Wang, C.-J. (Kaohsiung)

27 Extracorporeal Shockwave Therapy for Tendinopathy

Ko, J.-Y. (Kaohsiung/Xiamen); Wang, F.-S. (Kaohsiung)

42 Significance of Extracorporeal Shockwave Therapy in Fracture Treatment Haffner, N. (Vienna); Smolen, D. (Vienna/Pfäffikon); Dahm, F.; Schaden, W.; Mittermayr, R. (Vienna)

64 Local and Systemic Effects of Extracorporeal Shockwave Therapy on Bone Russo, S.; Servodidio, V.; Mosillo, G.; Sadile, F. (Naples)

70 Extracorporeal Shockwave Therapy and Sports-Related Injuries

Leal, C.; Berumen, E.; Fernandez, A.; Bucci, S.; Castillo, A. (Bogota, DC)

Section III: Cardiovascular Diseases

87 Preclinical and Clinical Application of Extracorporeal Shockwave for Ischemic Cardiovascular Disease

Yip, H.-K.; Lee, F.-Y.; Chen, K.-H.; Sung, P.-H.; Sun, C.-K. (Kaohsiung)

102 Mechanisms Underlying Extracorporeal Shockwave Treatment for Ischemic Cardiovascular Disease

Sun, C.-K.; Yip, H.-K. (Kaohsiung)

109 Effect of Extracorporeal Shockwave on Angiogenesis and Anti-Inflammation: Molecular-Cellular Signaling Pathways

Leu, S.; Huang, T.-H.; Chen, Y.-L.; Yip, H.-K. (Kaohsiung) 
Section IV: Urinary Diseases and Other Applications

117 Extracorporeal Shockwave Therapy Assisted Intravesical Drug Delivery Tyagi, P. (Pittsburgh, PA); Chuang, Y.-C. (Kaohsiung)

127 Application of Extracorporeal Shockwave Therapy on Erectile Dysfunction and Lower Urinary Tract Inflammatory Diseases

Wang, H.-J. (Kaohsiung)

Section V: Future Prospects of Shockwave Medicine

140 Current Applications and Future Prospects of Extracorporeal Shockwave Therapy

Sansone, V. (Milan); Frairia, R. (Torino); Brañes, M. (Santiago); Romeo, P. (Milan);

Catalano, M.G. (Torino); Applefield, R.C. (Milan)

158 Author Index

159 Subject Index 


\section{Foreword}

Welcome to volume 6 of Translational Research in Biomedicine, a monograph series dedicated to the dissemination of seminal information in contemporary biomedicine with a translational orientation.

This volume is designed to be a comprehensive reference for shockwave medicine, a relatively new clinical specialty in modern medicine. Originally established as the gold standard for the disintegration of kidney stones, Extracorporeal Shockwave Therapy (ESWT) has progressively evolved to a regenerative treatment modality, and is currently indicated for musculoskeletal disorders and nonskeletal diseases that include ischemic heart disease, diabetic foot ulcers, acute or chronic wounds, and burn lesions. Authored by a panel of international experts and in the spirit of translational medicine, this volume provides a succinct summary of the history and principles of ESWT, the documentations that substantiate the launch of this new clinical specialty, the myriad of physical and cellular or molecular mechanisms that underpin the effects of shockwave, and the future prospects and directions of ESWT.

I wish to express my deep appreciation to Professors Ching-Jen Wang, Wolfgang Schaden, and Jih-Yang Ko, who have taken time from their heavy clinical duties and research endeavors to make this timely volume on "Shockwave Medicine" a reality. I am indebted to the generous patronage of Chang Gung Medical Foundation, Taiwan, which reduces substantially the increasing financial constraints on scientific publication, and allows us to concentrate on publishing timely and crucial themes in translational medicine. I also wish to acknowledge the capable hands of Freddy Brian and Angela Hefti at S. Karger AG during the development and production of this volume. Last but not least, the publication of Translational Research in Biomedicine would not have been possible without the foresight, enthusiasm, and whole-hearted support of my dear friend, Dr. Thomas Karger.

Samuel H.H. Chan, Kaohsiung Series Editor 


\section{Preface}

Extracorporeal Shockwave Therapy (ESWT) was originally designed for the disintegration of kidney stones and has remained the gold standard for the treatment of urolithiasis because of its clinical success. In orthopedics, an incidental observation of osteoblast responses in bone after ESWT triggered the clinical use of this practice for musculoskeletal disorders. This change resulted in expanding ESWT from a destructive force for stone disintegration into a regenerative treatment modality. Advancements in research and technology further revealed additional mechanisms and pathways of action, and widened the indications of ESWT from musculoskeletal disorders to non-skeletal diseases such as ischemic heart disease, diabetic foot ulcers, acute or chronic wounds, and burn lesions. As such, ESWT has evolved into a multidisciplinary and multifunctional medical subspecialty.

Despite its clinical success, the exact mechanism of ESWT in biological tissue remains unknown. In 1997, Dr. Haupt [1] proposed 4 possible mechanisms for the actions of ESWT on tissue. In its physical phase, ESWT causes a positive pressure to generate absorption, reflection, refraction, and transmission of energy on tissues or cells. Additional studies demonstrated a negative pressure to induce the physical effects such as cavitation and increasing the permeability of cell membrane and ionization of biological tissues. Many signal transductions, including mechanotransduction signal pathway, the extracellular signal-regulated signal kinase, focal adhesion kinase signal pathway, and toll-like receptor 3 signal pathway to regulate gene expression, are activated. In its physical-chemical phase, ESWT stimulates cells to release biomolecules such as ATP to activate signal pathways. ESWT also alters the functions of ion channel in cell membrane and calcium mobilization in cells. In biological tissues, ESWT modulates angiogenesis (vWF, VEGF, eNOS, and PCNA), bone healing (BMP2, osteocalcin, alkaline phosphatase, DKK1 and IGF-1), anti-inflammatory (siCAM and sVCAM) and wound healing (Wnt3 and B-catenin). Furthermore, ESWT stimulates the shift of the macrophage phenotypes from M1 to M2 and increases Tcell proliferation. ESWT activates the toll-like receptor 3 signal pathway to modulate inflammation by controlling the expression of IL6-10 and improves the treatment in ischemic muscles. The future application of ESWT should be combined with advanced and innovative technology, including stem cell therapy, miRNA analysis, gene 
sequencing, and genomic medicine. This book follows the evolution of ESWT in medicine from the initial stage of destructive ESWT force for lithotripsy to regenerative effects in biological tissues. Section 1 covers the history of shockwave treatment and basic principles. Section 2 includes the application of ESWT in musculoskeletal disorders including osteonecrosis of the femoral head (hip), tendinopathy, fracture treatment, local and systemic effects of ESWT on bone and ESWT and sports related injuries. Section 3 explains the application of ESWT for cardiovascular diseases including preclinical and clinical application of ESWT for ischemic cardiovascular disease and mechanism underlying ESWT for ischemic cardiovascular disease and effects of ESWT on angiogenesis and anti-inflammation-molecular-cellular signaling pathways. Section 4 elaborated on the application of ESWT in urinary disease and other applications including ESWT-assisted intravesical drug delivery and erectile dysfunction and lower urinary tract inflammatory diseases. Section 5 presents the future prospects of shockwave medicine including current application and future procedures of ESWT.

We are grateful to Karger Publishers and to the Series Editor, Professor Samuel H.H. Chan for the opportunity to publish this volume. This book commemorates the two-year anniversary of the Center for Shockwave Medicine and Tissue Engineering at Kaohsiung Chang Gung Memorial Hospital. This book contains the most complete and up-to-date ESWT-related data and documents with worldwide implications. We are committed to pursue ESWT-related research and new discovery with innovative procedures along with the new indications in shockwave medicine.

Ching-Jen Wang, Kaohsiung Wolfgang Schaden, Vienna Jih-Yang Ko, Kaohsiung

\section{Reference}

1 Haupt G: Use of extracorporeal shock waves in the treatment of pseudarthrosis, tendinopathy and other orthopedic diseases. J Urol 1997;158:4-11. 
